\title{
Sur les traces de l'or brésilien dans les monnayages européens
}

Jean-Noël Barrandon

\section{(2) OpenEdition}

\section{Journals}

Édition électronique

URL : http://journals.openedition.org/ress/216

DOI : $10.4000 /$ ress.216

ISSN : 1663-4446

Éditeur

Librairie Droz

Édition imprimée

Date de publication : 1 juillet 2007

Pagination : 99-106

ISBN : 978-2-600-01155-6

ISSN : 0048-8046

Référence électronique

Jean-Noël Barrandon, «Sur les traces de l'or brésilien dans les monnayages européens », Revue européenne des sciences sociales [En ligne], XLV-137 | 2007, mis en ligne le 01 juillet 2010, consulté le 30 avril 2019. URL : http://journals.openedition.org/ress/216 ; DOI : 10.4000/ress.216 


\section{SUR LES TRACES DE L'OR BRÉSILIEN DANS LES MONNAYAGES EUROPÉENS}

Il est évident que l'or brésilien a joué un rôle important dans l'économie européenne. En effet, ce dernier représentait plus de $40 \%$ de la production mondiale (Ridgeway, fig. $\mathrm{n}^{\circ} 1$ ) et l'ensemble Colombie-Brésil $70 \%$ du flux total ; cet or sud-américain restera important jusqu'à l'arrivée sur le marché de l'or russe durant le premier quart du XIX ${ }^{\mathrm{e}}$ siècle $^{1}$.

De nombreux historiens, de Braudel à Morineau, ont étudié ces phénomènes, mais leurs quantifications se heurtent aux lacunes dans les données statistiques, commerciales ou monétaires. Comme l'écrivait le grand historien Carlo Cipolla : «We know where the American treasure came from and when it reached Spain. To find out where and when it went from there and the uses it was put to is an intriguing and complex historical problem...» ${ }^{2}$

Notre propos est donc de présenter une méthode, appliquée ici à l'or du Brésil, qui permet de «tracer» le métal jaune sud-américain et donc de quantifier son importance dans les économies européennes.

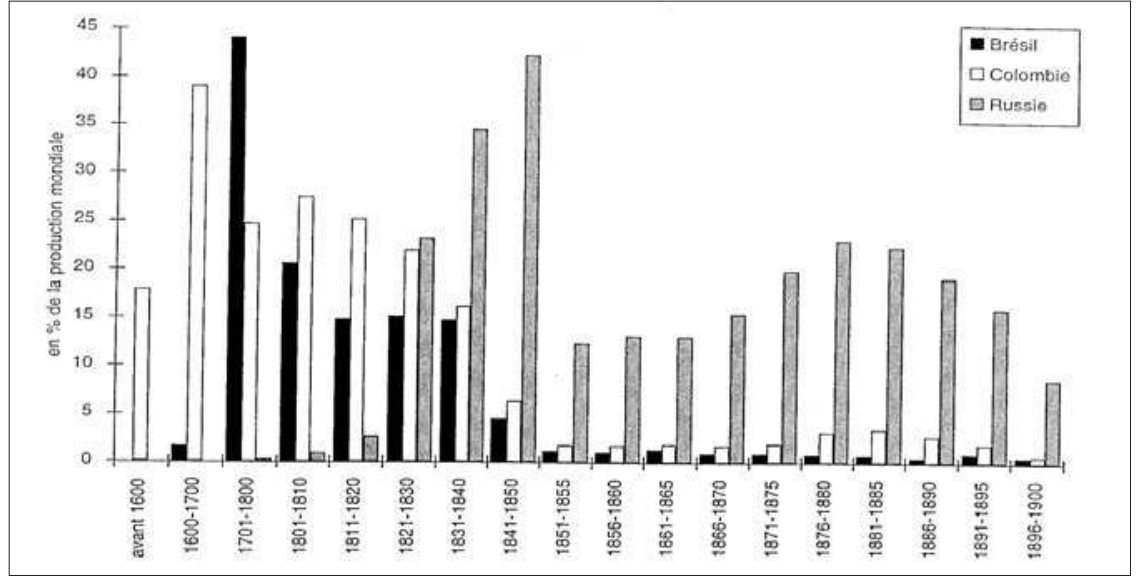

Figure 1: La part de l'or du Brésil, de Colombie et de Russie dans la production mondiale 1600-1900

\footnotetext{
Ridgeway (1929).

2 Cippola (1989).
} 
Cette méthode est basée sur l'utilisation d'un ou de plusieurs éléments chimiques dont l'analyse permet de suivre le minerai depuis son extraction jusqu'à son utilisation monétaire. Cet élément chimique appelé «marqueur» doit répondre à différents critères :

1. Il doit être spécifique du minerai que l'on veut tracer.

2. Il doit être dosable, de manière non destructive, dans les monnaies et cela avec une bonne précision (de l'ordre de $5 \%$ relatif au moins).

3. Il ne doit pas être affecté par les traitements minéralurgiques et métallurgiques que subissent le minerai puis le métal.

4. Il doit être en quantité beaucoup plus importante dans le minerai que l'on veut tracer que dans le métal dans lequel il va être refondu ; ce point est fondamental et peut conduire, s'il n'est pas respecté, à des conclusions erronées voire complètement fausses.

Voyons, à titre d'exemple, le cas de l'or «traceur» utilisé par A. et J. Gordus en 1971, afin de suivre l'argent du Potosi en Europe ${ }^{3}$. L'or répondait très bien aux trois premiers critères mais malheureusement ses teneurs dans l'argent potosien sont beaucoup plus faibles que dans les ors européens utilisés avant la découverte du Cerro-Rico (fig. $\mathrm{n}^{\circ}$ 2) et, de ce fait, une faible arrivée d'argent du Potosi en Espagne ne change pratiquement pas la teneur en $A u$ dans l'argent espagnol ; néanmoins, certaines monnaies espagnoles (celles dans l'ellipse) contiennent peu d'or et sont donc frappées avec de l'argent cent pour cent potosien.

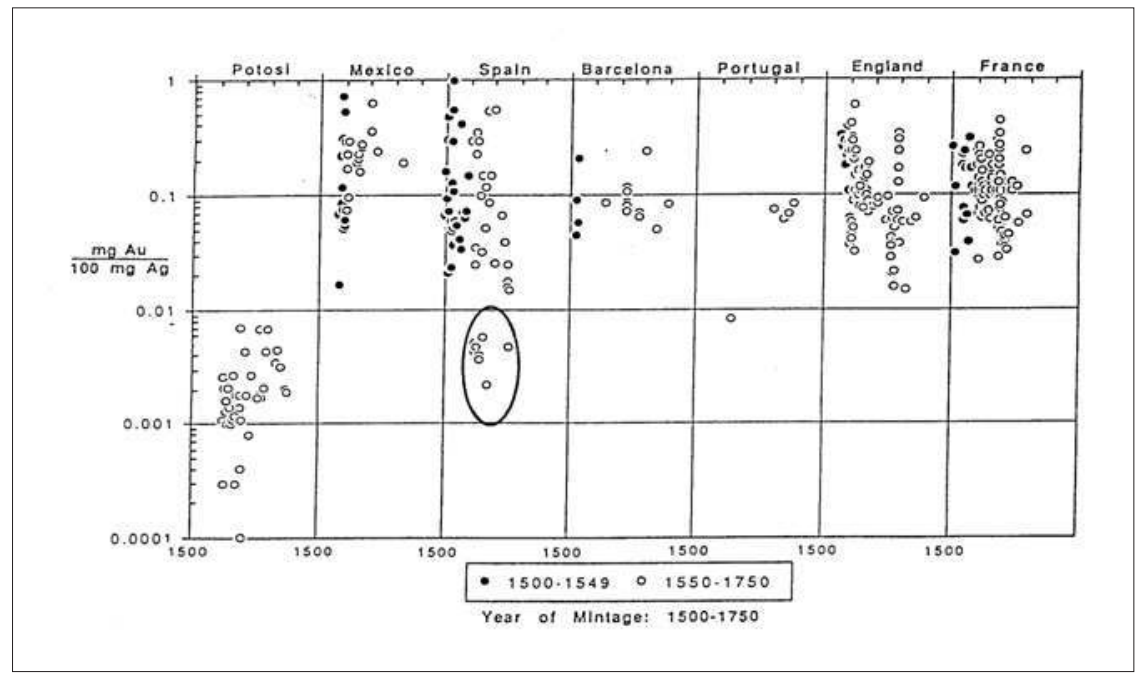

Figure 2: Les teneurs en or dans l'argent monétaire du Potosi, du Mexique et de l'Europe

3 Gordus, Le Roy Ladurie et Richet (1972). 
Ayant sous-estimé les résultats de ces quelques pièces, les auteurs allaient même jusqu'à remettre en question l'importance des arrivages d'argent du Potosi dans le monnayage d'Espagne ; cela montre bien l'importance de ce critère.

Dans le cas de l'or brésilien, une des spécificités des sables aurifères de la région de Minas Gerais est de contenir des quantités importantes de palladium (tab. $\left.\mathrm{n}^{\circ} 1\right)$; ce palladium (Pd) est-il un bon «traceur» de l'or brésilien ? Oui, car il répond aux critères définis précédemment.

Tableau 1: Analyses d'or en grains provenant du Brésil

\begin{tabular}{|l|c|c|c|}
\hline & Au en $\%$ & Ag en $\%$ & Pd en $\%$ \\
\hline Pépite près de Sabara & 85,98 & 4,17 & 9,85 \\
\hline Pépite de Taguaril (Minas Gerais) & 91,06 & & 8,12 \\
\hline Pépites analysées par La Monnaie & 88,90 & & 11,1 \\
de Rio & 92,00 & & 7,30 \\
\hline
\end{tabular}

Source : de Launay, 1895.

- Le palladium est un élément chimique de «la mine du platine», donc qui suit l'or dans les procédés métallurgiques mis en œuvre pour purifier le minerai.

- Il est contenu à des teneurs de l'ordre de 1000 ppm dans les monnaies frappées par les ateliers brésiliens, alors qu'il n'est qu'à des teneurs de l'ordre de 20 ppm dans les monnaies portugaises, anglaises et françaises frappées avant $1702\left(\right.$ tab. $\left.n^{\circ} 2\right)$.

- Enfin, le palladium est facilement dosable par analyse par activation protonique au cyclotron, méthode non destructive (tab. $\left.\mathrm{n}^{\circ} 3\right)^{4}$.

Afin de valider notre «marqueur», nous avons étudié l'évolution de la teneur en palladium dans les monnaies frappées pour l'atelier de Lisbonne de 1450 à 1800 (fig. $\mathrm{n}^{\circ}$ 3). La monnaie frappée en 1702 à la fin du règne de Pierre II (16821706) permet d'observer une augmentation significative de la teneur en $\mathrm{Pd}$ (125 ppm par rapport à 13 ppm, soit dix fois plus), prouvant de manière indiscutable l'arrivée, à partir de cette date, de l'or du Brésil, et de calculer la proportion d'or brésilien dans la monnaie.

\section{L'or brésilien dans les monnaies françaises d'après le palladium}

A la différence de l'or portugais qui, au XVIII ${ }^{\mathrm{e}}$ siècle, provient quasi exclusivement du Brésil, l’or français a plusieurs origines :

\footnotetext{
4 Barrandon (1978).
} 
Tableau 2: Le palladium dans les monnaies européennes antérieures à 1702

\begin{tabular}{|c|c|c|c|c|c|}
\hline \multicolumn{2}{|c|}{ Monnaies portugaises } & \multicolumn{2}{|c|}{ Monnaies anglaises } & \multicolumn{2}{|c|}{ Monnaies françaises } \\
\hline Année & Pd en ppm & Année & Pd en ppm & Année & Pd en ppm \\
\hline $\begin{array}{c}1460 \\
1460 \\
1460 \\
1460 \\
1508 \\
1539 \\
1539 \\
1539 \\
1568 \\
1568 \\
1589 \\
1648 \\
1690 \\
\end{array}$ & $\begin{array}{c}2,5 \\
34 \\
4 \\
21 \\
8 \\
7 \\
4 \\
2 \\
13 \\
21 \\
2,4 \\
3,7 \\
18 \\
\\
\\
\\
\\
13 \mathrm{ppm} \\
10 \mathrm{ppm}\end{array}$ & $\begin{array}{l}1632 \\
1695 \\
1695 \\
1702 \\
1702\end{array}$ & $\begin{array}{c}39 \\
27 \\
13 \\
24 \\
18 \\
\\
\\
23,5 \mathrm{ppm} \\
9 \mathrm{ppm}\end{array}$ & $\begin{array}{l}1660 \\
1669 \\
1680 \\
1693 \\
1694 \\
1694 \\
1701 \\
1702\end{array}$ & $\begin{array}{c}10 \\
25 \\
25 \\
19 \\
36 \\
20 \\
21,5 \\
22 \\
22,3 \mathrm{ppm} \\
7 \mathrm{ppm}\end{array}$ \\
\hline
\end{tabular}

Tableau 3: Résultats d'analyse sur trois monnaies d'or frappées respectivement au Brésil, au Portugal et en France

\begin{tabular}{|l|l|l|l|}
\hline Réf. Mon. & B. N. 1056 & B.N. 3045 & B.N. F 20 \\
$\begin{array}{l}\text { Dénomination } \\
\text { Atelier }\end{array}$ & Moeda & Cruzado & Demi-Louis \\
$\begin{array}{l}\text { Souverain } \\
\text { Date }\end{array}$ & B (Bahia) & Lisbonne & Paris \\
\hline Au en V $\%$ & Pierre II & Louis XV \\
Ag en \% & 1726 & 1690 & 1725 \\
Cu en \% & 89,9 & 91,0 & 89,2 \\
& 3,8 & 7,35 & 6,25 \\
\hline Sn (étain) & 6,0 & 1,47 & 3,89 \\
Sb (antimoine) & \multicolumn{3}{|l|}{ Eléments-traces en ppm } \\
Zn (zinc) & 15 & 150 & 326 \\
Fe (fer) & 30 & 20 & 83 \\
Pb (plomb) & 150 & 14,5 & 141 \\
Pd (palladium) & 180 & 470 & 2700 \\
Pt (platine) & 1420 & 18 & 392 \\
Hg (mercure) & 74 & 960 & 270 \\
Ti (titane) & 550 & 190 & 2670 \\
Te (tellure) & 1,3 & 1 & $/$ \\
\end{tabular}




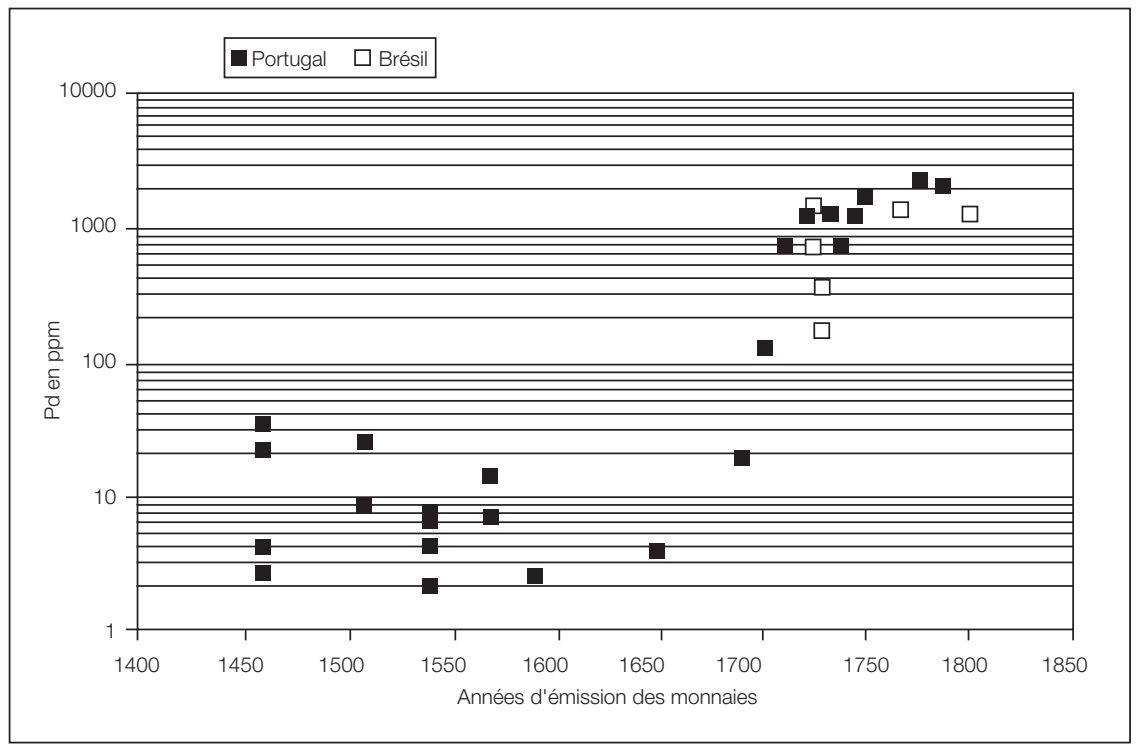

Figure 3: Le palladium dans les monnaies frappées au Brésil et au Portugal

- les pièces anciennes refondues,

- l'or du Brésil via le déficit portugais avec la France,

- l'or brésilien ayant transité par d'autres pays (par exemple, l'Angleterre).

Les teneurs en palladium dans les monnaies françaises sont données par le graphique, en échelle semi-logarithmique, de la fig. $\mathrm{n}^{\circ} 4$.

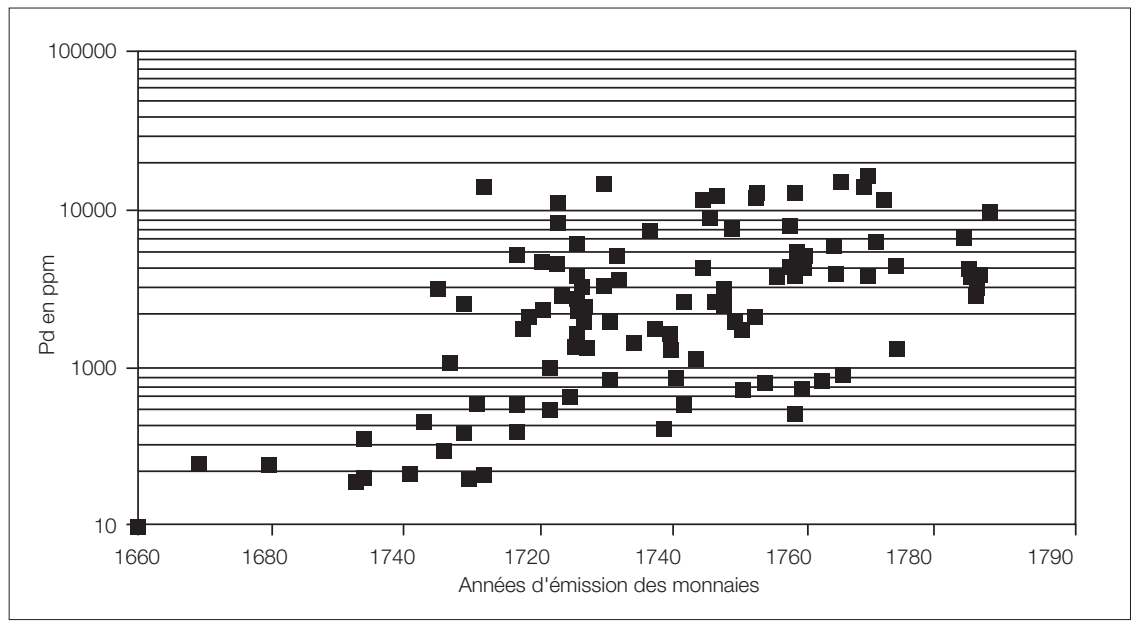

Figure 4: Le palladium dans les monnaies françaises de 1680 à 1785 
Le passage de la teneur en palladium au pourcentage d'or «brésilien» se fait par le calcul suivant :

$$
\% \text { d'or brésilien }=[(x-20) / 1115] \times 100
$$

dans lequel $x$ représente la teneur en palladium dans la monnaie en ppm ; 20 la teneur moyenne en palladium dans l'or français avant l'arrivée de l'or brésilien ; 1115 la teneur moyenne en palladium dans l'or du Brésil ; cette valeur a été obtenue en faisant la moyenne des teneurs obtenues sur les monnaies frappées au Brésil et celles frappées à Lisbonne au début du XVIII ${ }^{\mathrm{e}}$ siècle.

A titre d'exemple, si $\mathrm{x}=580 \mathrm{ppm}$, le pourcentage d'or sera :

$$
\%=[(580-20) / 1115] 100
$$

\section{Sur les traces de l'or brésilien dans les monnayages français}

A partir des valeurs individuelles, nous avons calculé des pourcentages moyens (décennaux sauf pour les années 1726,1727 et 1785 ) (fig. $\mathrm{n}^{\circ} 5$ ) ; ce qui nous a permis, à partir des statistiques des frappes annuelles ${ }^{5}$, d'estimer les quantités en millions de livres tournois d'or brésilien monnayé.

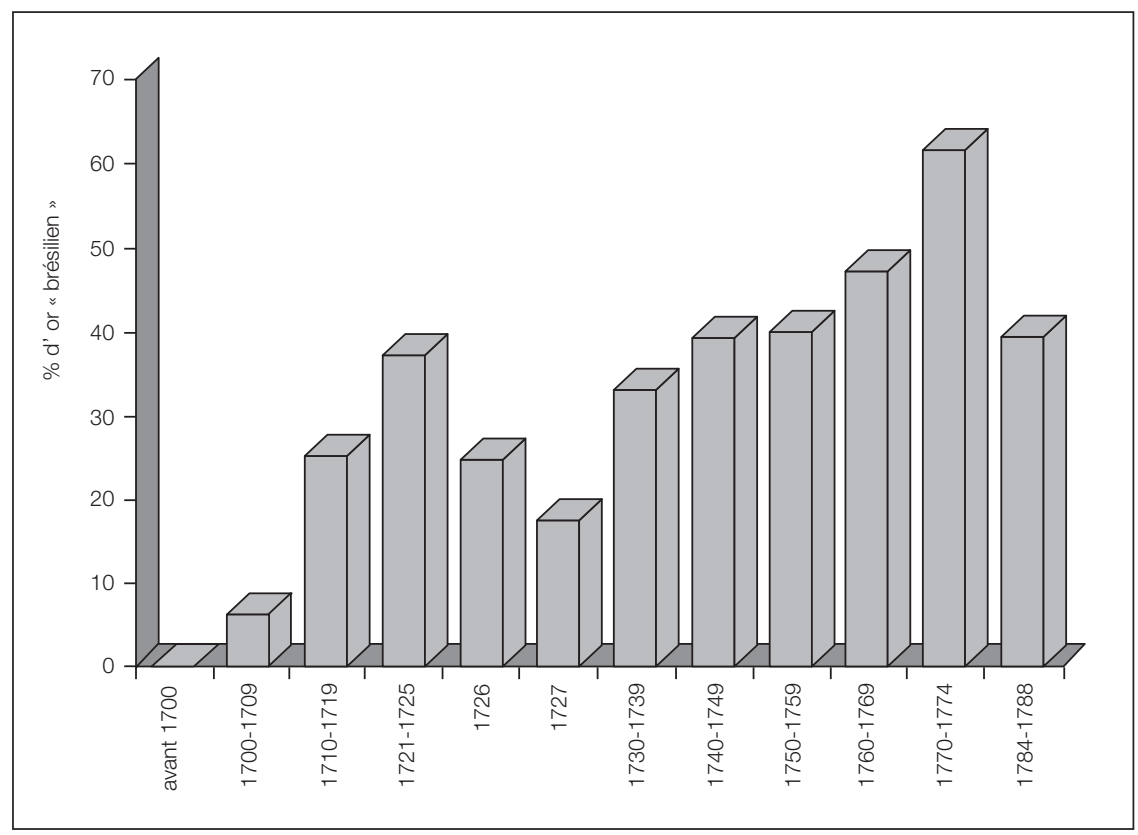

Figure 5: Pourcentage d'or brésilien dans le monnayage français de 1700 à 1785

5 Morrisson, Barrandon et Morrisson (1999), p. 55 et suiv. 
Deux dates présentent un intérêt tout à fait particulier : les refontes de 1726 et de 1785 .

\section{La refonte de $\mathbf{1 7 2 6}$}

A partir des 19 exemplaires analysés, nous avons calculé une proportion de $25,2 \%$ d'or brésilien. Le montant des frappes étant de 160,5 millions de livres tournois, on en déduit un montant d'or brésilien d'environ 40 millions de livres tournois, valeur qui représente la totalité de l'or brésilien rentré en France durant la période 1703-1725.

\section{La refonte de $\mathbf{1 7 8 5}$}

Pour les frappes de 1786, on trouve $30 \%$ d'or brésilien ; ainsi, sur les 708 millions d'or frappé durant les années 1786-1788, 212 millions sont imputables aux entrées d'or du Brésil pour la période 1783-1785; chiffre qui est à mettre en parallèle avec les 218 millions d'excédent commercial cumulé avec le Portugal.

\section{CONCLUSIONS ET PERSPECTIVES}

Nous venons de démontrer que le palladium est un «bon traceur» de l'or brésilien puisqu'il permet de quantifier les arrivées de ce nouveau métal en Europe. La question qui se pose est : peut-on généraliser ce type de recherche? En d'autres termes, peut-on étudier par la même méthode, l'argent du Potosi, du Mexique, l'or du Pérou, de la Colombie ou de la Russie, etc. ? La réponse est non. Néanmoins, certains cas sont favorables, citons l'argent du Potosi avec le traceur indium et l'or de Colombie avec le traceur platine, dont nous voudrions dire ici deux mots avant de conclure.

Si l'on regarde l'évolution comparée des teneurs en palladium et en platine dans l'or frappé en Angleterre de 1690 à 1760 (fig. n 6), on voit que, avant l'ar-

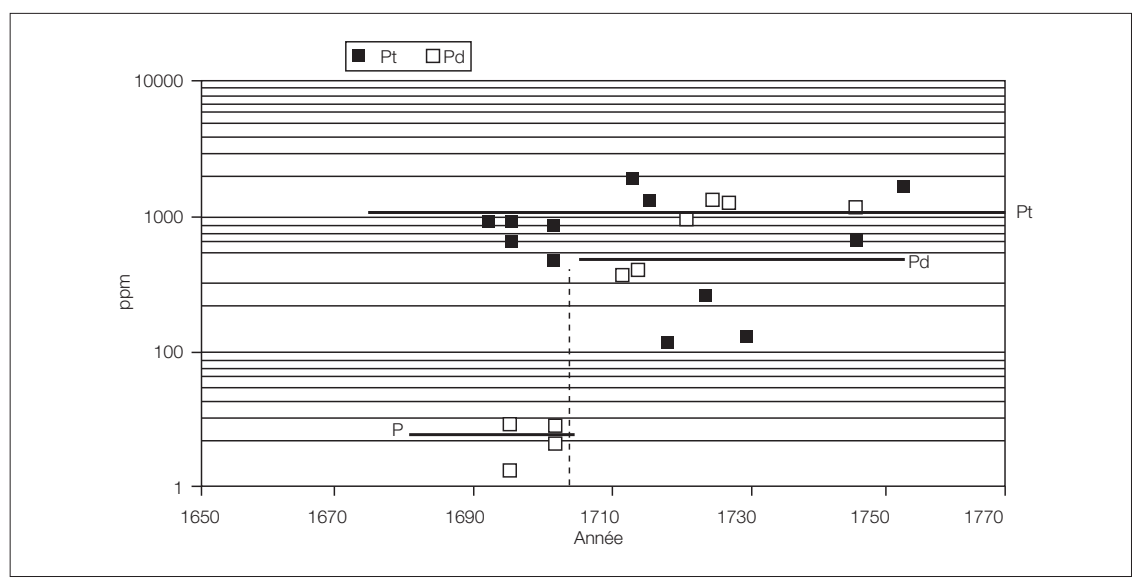

Figure 6: Platine et palladium dans l’or frappé en Angleterre de 1690 à 1760 (échelle semi-log) 


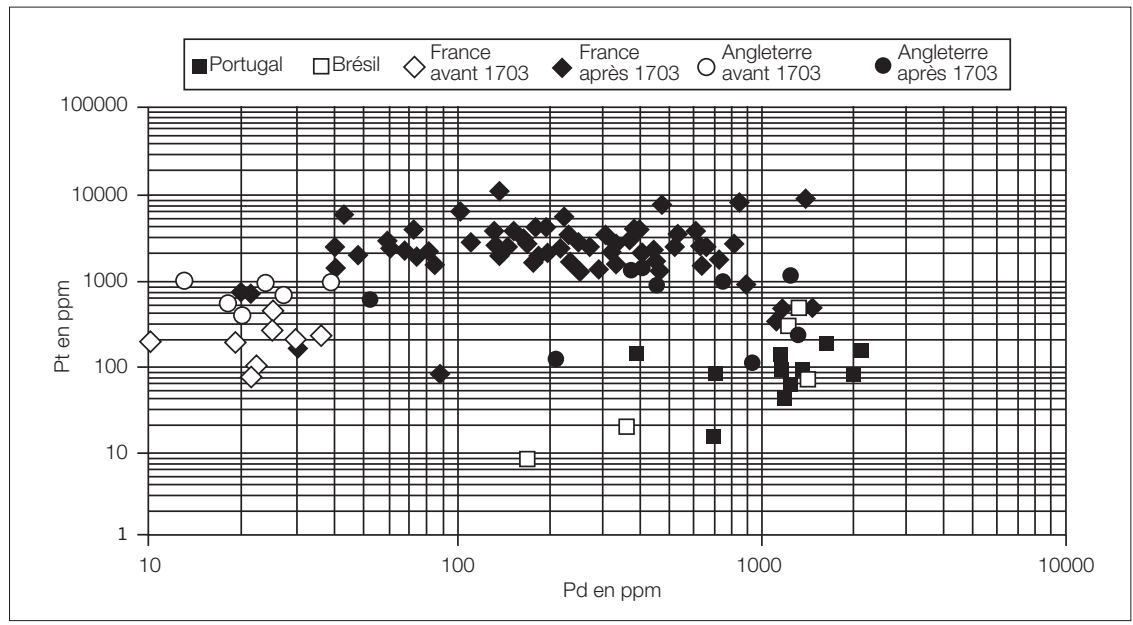

Figure 7: Platine et palladium dans l'or frappé avant et après 1703 au Brésil, au Portugal, en Angleterre et en France (échelle log-log)

rivée de l'or brésilien, l'or «anglais » contient des teneurs importantes en platine. Il en est de même pour la France (fig. ${ }^{\circ} 7$ ). Cet «or platiné», d'où provient-il ? Deux possibilités : la Russie et la Colombie, connues pour leurs placeurs «d'or platiné». La Russie doit être écartée, car la découverte de l'or de l'Oural date de 1745 et ce site n'a été exploité qu'à partir de 1752; par contre, l'or de Colombie a été exploité dès les premières décennies du XVII ${ }^{\mathrm{e}}$ siècle, le platine est donc un bon «traceur» de l'or colombien pour la période XVII ${ }^{\mathrm{e}}-\mathrm{XVIII}{ }^{\mathrm{e}}$ siècle et permet d'estimer les quantités d'or colombien arrivant en Europe via l'Espagne ${ }^{6}$.

\section{BIBLIOGRAPHIE}

Barrandon, Jean-Noël, «Utilisation des réactions nucléaires induites par des protons pour l'étude des monnaies d'argent et d'or», Revue d'Archéométrie, 1978, pp. 105-120.

Cipolla, Calo M., «American treasure and the florentine coinage, in the sixteenth century», in E.H.F. Van Cauvenbergh (ed.), Precious metals, coinage and the changes of monetary structures in Latin-America, Europe and Asia, Leuven University Press, 1989, pp. 69-75.

Gordus, Adon et Jeanne, Emmanuel Le Roy Ladurie et Denis Richet, «Le Potosi et la physique nucléaire », Annales E.S.C., 1972, pp. 1235-1256.

Morrisson, Christian, Barrandon, Jean-Noël et Morrisson, Cécile, «Or du Brésil, monnaie et croissance en France au XVIII ${ }^{\mathrm{e}}$ siècle, in Cahiers Ernest-Babelon, n ${ }^{\circ}$ 7, CNRS éditions, 1999.

Ridgeway, R.H., «Summarized data of silver production US Bureau of Mines», Economic paper, $\mathrm{n}^{\circ} 6,1929$, Washington.

\footnotetext{
6 Morrisson, Barrandon, et Morrisson, op. cit., p. 118 et suiv.
} 